\title{
Valuations and Polarity
}

\author{
Jim Lawrence \\ Department of Mathematical Sciences, George Mason University, \\ Fairfax, VA 22030, USA
}

\begin{abstract}
Given a collection $\mathscr{A}$ of convex polytopes, let $\tau(\mathscr{A})$ denote the set of all convex transversals of $\mathscr{A}$. If $\mathscr{A}$ and $\mathscr{B}$ are two such collections, of finite cardinality, then there is a simple, arithmetical condition which holds precisely when $\tau(\mathscr{A})=$ $\tau(\mathscr{B})$. Another such condition, involving what we call the "Sallee-Shephard mapping," characterizes those pairs $\mathscr{A}$ and $\mathscr{B}$ for which $\tau(\tau(\mathscr{A}))=\tau(\mathscr{B})$.

As these results are established, several distributive lattices involving convex sets are introduced, and relationships between their valuation modules are determined. In particular, it is proven that the Sallee-Shephard mapping is an isomorphism of the additive, abelian group of simple functions generated by the characteristic functions of the open, convex sets and that generated by those of the closed, convex sets.
\end{abstract}

\section{Introduction}

The writing of this paper began as an attempt to exploit the notion of "polarity" of convex sets in the theory of valuations on convex polyhedra. In the first sections we give results without explicit reference to the connection with polarity; but in many cases a simpler proof (of a possibly weaker result) can be obtained by appealing to later results more directly concerned with polarity. The final section of this paper is largely self-contained and the reader might wish to glance at this section early on.

In Sections 2, 4, and 5 various distributive lattices are described. Among these are two familiar ones: $L_{\mathrm{o}}$ and $L_{\mathrm{c}}$, the lattices of finite unions of open and closed, respectively, convex sets in $\mathscr{R}^{d}$. Three less-familiar ones are: $\Phi$, the lattice of "complete families of convex transversals;" and $\hat{L}_{\mathrm{o}}$ and $\hat{L}_{\mathrm{c}}$, the "polar lattices of open (closed) convex sets."

The valuation modules of $L_{\mathrm{o}}, \hat{L}_{\mathrm{o}}, L_{\mathrm{c}}$, and $\hat{L}_{\mathrm{c}}$ are isomorphic. Indeed, we show that those of $L_{\mathrm{o}}$ and of $\hat{L}_{\mathrm{o}}$ are isomorphic to $\mathbb{Z} \oplus S_{\mathrm{o}}$, where $S_{\mathrm{o}}$ is the group 
( $\mathbb{Z}$-module) of simple functions generated by the characteristic functions of open, convex sets. Similarly, those of $L_{\mathrm{c}}$ and $\hat{L}_{\mathrm{c}}$ are isomorphic to $\mathbb{Z} \oplus S_{\mathrm{c}}$, where $S_{\mathrm{c}}$ is the group generated by the characteristic functions of the closed, convex sets. In Section 6 it is shown that $S_{\mathrm{o}}$ and $S_{\mathrm{c}}$ are connected by an isomorphism, the "Sallee-Shephard mapping."

In Section 3 an arithmetical condition is given for two finite collections $\mathscr{A}$ and $\mathscr{B}$ of convex sets to possess the same convex transversals. The Sallee-Shephard mapping is introduced and used in Section 6 to shed further light on this topic.

The paper makes use of fundamental facts concerning convex sets and polytopes, which may be found in [7], [15], and [19]. The inspirational papers of Hadwiger [8], Klee [11], and Rota [16] provide bacground on the Euler characteristic in a suitable setting, and on valuations, more generally. The paper of McMullen and Schneider [14] provides a useful survey of the use of valuations in convexity. The papers of Geissinger [2] provide the basic facts concerning the valuation modules and valuation rings of distributive lattices. The papers of Groemer [3]-[6] contain more related material.

\section{Complete Families of Transversals}

Given a family $\mathscr{K}$ of convex subsets of $\mathscr{R}^{d}$, let $\tau(\mathscr{K})$ denote the family of convex transversals of $\mathscr{K}$, so that $\tau(\mathscr{K})=\left\{T \subseteq \mathscr{R}^{d}: T \cap \mathscr{K} \neq \varnothing\right.$ for each $\left.K \in \mathscr{K}\right\}$. We will call sets of the form $\tau(\mathscr{K})$ complete families of transversals, and we will denote by $\Phi$ the collection of all complete families of transversals.

It follows from the formula $\bigcap_{\lambda \in \Lambda} \tau\left(\mathscr{K}_{\lambda}\right)=\tau\left(\bigcup_{\lambda \in \Lambda} \mathscr{K}_{\lambda}\right)$ that the intersection of any collection of complete families of transversals is again in $\Phi$. The family of all convex sets in $\mathscr{R}^{d}$ is $\tau(\varnothing)$, an element of $\Phi$. It follows that $\Phi$, as a set partially ordered by inclusion, is a complete lattice.

The meet operation on $\Phi$ is intersection. The join of a collection $\mathscr{K}_{\lambda}(\lambda \in \Lambda)$ of elements of $\Phi$ is $\varphi\left(\bigcup_{\lambda \in \Lambda} \mathscr{K}_{\lambda}\right)$, where for any collection $\mathscr{K}$ of convex sets,

$$
\phi(\mathscr{K})=\tau(\tau(\mathscr{K}))=\bigcap_{\substack{\mathscr{T} \in \Phi, \mathscr{T} \subseteq \mathscr{T}}} \mathscr{T}
$$

Note that $\tau$, when restricted to $\Phi$, is a dual automorphism of $\Phi$. It is worthwhile to observe that a criterion for inclusion of a family $\mathscr{T}$ in $\Phi$ is that for each convex set $K \notin \mathscr{T}$, there is a convex transversal $T$ of $\mathscr{T}$ such that $K \cap T=\varnothing$.

The foregoing paragraphs describe some essential pieces of the structure one obtains by considering the binary relation " $K$ has nonempty intersection with $T$ " on the family of convex sets in $\mathscr{R}^{d}$ and using this relation to obtain a "polarity," as in pp. 122-125 of [1].

In this section we study the structure of $\Phi$. In particular we show that it is distributive.

We call a set $H \subseteq \mathscr{R}^{d}$ a half-space if both $H$ and its complement, $\mathscr{R}^{d} \sim H$, are convex. (Note that a half-space may be neither closed nor open. Our half-spaces are called "hemispaces" by Jamison [10].) Let $\mathscr{H}$ denote the collection of all 
half-spaces in $\mathscr{R}^{d}$, partially ordered by inclusion. Let $\hat{\mathscr{H}}$ denote the collection of all upper semi-ideals of $\mathscr{H}$; i.e., a subset $\mathscr{J} \subseteq \mathscr{H}$ is an element of $\hat{\mathscr{H}}$ if, for half-spaces $H_{1}$ and $H_{2}, H_{1} \in \mathscr{J}$ and $H_{2} \supseteq H_{1}$ imply $H_{2} \in \mathscr{J}$. Clearly, $\hat{\mathscr{H}}$ is a completely distributive lattice. Its operations are intersection and union.

For $\mathscr{T} \in \Phi$, let $\gamma(\mathscr{T})=\mathscr{T} \cap \mathscr{H}$. This is a function $\gamma: \Phi \rightarrow \hat{H}$, since elements $\mathscr{T}$ of $\Phi$ are upper simi-ideals in the lattice of all subsets of the set of convex sets in $\mathscr{R}^{d}$, ordered by inclusion.

Theorem 1. The function $\gamma: \Phi \rightarrow \hat{\mathscr{H}}$ is an isomorphism of partially ordered sets (and, therefore, of complete lattices).

Proof. We first show that for complete families of transversals $\mathscr{T}_{1}$ and $\mathscr{T}_{2}$, $\mathscr{T}_{1} \subseteq \mathscr{T}_{2}$ if and only if $\gamma\left(\mathscr{T}_{1}\right) \subseteq \gamma\left(\mathscr{T}_{2}\right)$. If $\mathscr{T}_{1} \subseteq \mathscr{T}_{2}$, it is immediate that $\gamma\left(\mathscr{T}_{1}\right)=\mathscr{T}_{1} \cap$ $\mathscr{H} \subseteq \mathscr{T}_{2} \cap \mathscr{H}=\gamma\left(\mathscr{T}_{2}\right)$. Suppose $\mathscr{T}_{1} \nsubseteq \mathscr{T}_{2}$. Let $K$ be an element of $\mathscr{T}_{1} \sim \mathscr{T}_{2}$. Since $K \notin \mathscr{T}_{2}$, there is a transversal $T$ of $\mathscr{T}_{2}$ such that $K \cap T=\varnothing$. By a result of Hammer [9] there is a half-space $H$ such that $K \subseteq H$ and $T \subseteq \mathscr{R}^{d} \sim H$. Then $H \in \mathscr{T}_{1} \sim \mathscr{T}_{2}$, so $\gamma\left(\mathscr{T}_{1}\right) \notin \gamma\left(\mathscr{T}_{2}\right)$.

It remains to show that $\gamma$ is surjective. Suppose $\mathscr{J} \in \hat{\mathscr{H}}$. Let $\mathscr{T}=\left\{K \subseteq \mathscr{R}^{d}: K\right.$ is convex, and for each transversal $T$ of $\mathscr{F}$ it is true that $T \cap K \neq \varnothing\}$; i.e., $\mathscr{T}=\varphi(\mathscr{F})$. Then $\mathscr{T} \in \Phi$. We need only show that $\gamma(\mathscr{T})=\mathscr{H}$. Clearly, $\mathscr{J} \subseteq \mathscr{T}$, so $\mathscr{J} \subseteq \mathscr{T} \cap \mathscr{H}=$ $\gamma(\mathscr{T})$. We verify the reverse inclusion. Suppose that $H$ is a half-space which is not in $\mathscr{g}$. We must show that $H$ is not in $\mathscr{T}$. Let $T=\mathscr{R}^{d} \sim H$. Then $T$ is a transversal of $\mathscr{J}$, for if $J \in \mathscr{J}$ then, since $H \notin \mathscr{Z}, J \nsubseteq H$, so $J \cap T \neq \varnothing$. Since $T \cap H=\varnothing$ it follows that $H \notin \mathscr{T}$.

Corollary. The lattice $\Phi$ is completely distributive.

Let $\Phi_{\mathrm{p}}$ denote that subset of $\Phi$ consisting of all complete families of transversals which are of the form $\tau(\mathscr{K})$, where $\mathscr{K}$ is a finite family of convex polytopes.

Theorem 2. The subset $\Phi_{\mathrm{p}} \subseteq \Phi$ is a sublattice of $\Phi$ (in the finitary sense). The restriction of $\tau$ to $\Phi_{\mathrm{p}}$ is a dual automorphism of $\Phi_{\mathrm{p}}$.

Proof. Temporarily denote by $\Phi^{\prime}$ the sublattice of $\Phi$ generated by elements of the form $\tau(\{p\})$, where $p \in \mathscr{R}^{d}$. Note that such elements are fixed by $\tau$, so that $\tau$ maps $\Phi^{\prime}$ to itself. It remains only to show that $\Phi^{\prime}=\Phi_{\mathrm{p}}$.

Suppose $\left\{v_{1}, \ldots, v_{n}\right\} \subseteq \mathscr{R}^{d}$ and $P=\operatorname{conv}\left\{v_{1}, \ldots, v_{n}\right\}$. Then $\tau\left(\left\{\left\{v_{1}\right\}, \ldots,\left\{v_{n}\right\}\right\}\right)$ consists of precisely those convex sets which contain $P$; clearly, this is $\varphi(\{P\})$. From the equality $\varphi(\{P\})=\tau\left(\left\{\left\{v_{1}\right\}, \ldots,\left\{v_{n}\right\}\right\}\right)$ follows $\tau(\{P\})=\tau(\varphi(\{P\}))=$ $\varphi\left(\left\{\left\{v_{1}\right\}, \ldots,\left\{v_{n}\right\}\right\}\right)$. We see that (i) the join $\varphi\left(\left\{\left\{v_{1}\right\}, \ldots,\left\{v_{n}\right\}\right\}\right)$ of the elements $\varphi\left(\left\{\left\{v_{i}\right\}\right\}\right)=\tau\left(\left\{\left\{v_{i}\right\}\right\}\right)$ (for $\left.i \in[n]\right)$ is $\tau(\{P\})$, and (ii) $\tau(\{P\}) \in \Phi^{\prime}$ (for any convex polytope $P$ ).

If $\mathscr{K}=\left\{P_{1}, \ldots, P_{m}\right\}$ is a finite collection of convex polytopes, so that $\tau(\mathscr{K}) \in \Phi_{\mathrm{p}}$, then $\tau(\mathscr{K})=\tau\left(\left\{P_{1}\right\}\right) \cap \cdots \cap \tau\left(\left\{P_{m}\right\}\right)$, which, utilizing (ii) above, is in $\Phi^{\prime}$. Then $\Phi_{\mathrm{p}} \subseteq \Phi^{\prime}$. The reverse inclusion follows easily from the fact that, since $\Phi$ is distributive, each element of the lattice $\Phi^{\prime}$ can be written (finitarily) as an intersection of joins of elements of the form $\tau(\{p\})$, so it is of the form $\tau(\mathscr{K})$. 


\section{A Consequence of the Equality $\tau\left(\mathscr{K}_{1}\right)=\tau\left(\mathscr{K}_{2}\right)$}

Our main objective in this section is the proof of Theorem 3 , below. In case $\mathscr{K}_{1}$ and $\mathscr{K}_{2}$ are finite families of convex sets and $\tau\left(\mathscr{K}_{1}\right)=\tau\left(\mathscr{K}_{2}\right)$, this result describes an equality involving $\mathscr{K}_{1}$ and $\mathscr{K}_{2}$ which must hold in the valuation ring of the lattice of finite unions of convex sets. The proof roughly mimics (through polarity) part of an argument used by Groemer in his proof of the existence of an Euler characteristic. (See [3].)

A consequence of Theorem 3 for "clustered families" of convex sets, introduced in [12], is also described.

If $K$ and $G$ are convex sets, let $\delta(K, G)=\{a g+(1-a) x: 0 \leq a<1, g \in G$, and $x \in K\}$. Clearly, this is also a convex set, and $K \subseteq \delta(K, G) \subseteq \operatorname{conv}(K \cap G)$.

It is not difficult to establish that, if $\mathscr{H}$ and $\mathscr{G}$ are families of convex sets, then

$$
\delta\left(\operatorname{conv} \bigcup_{K \in \mathscr{K}} K, \text { conv } \bigcup_{G \in \mathscr{G}} G\right)=\operatorname{conv} \bigcup_{\substack{K \in \mathscr{K} \\ G \in \mathscr{G}}} \delta(K, G)
$$

Also, if $K, G_{1}$, and $G_{2}$ are convex, then $\delta\left(\delta\left(K, G_{1}\right), G_{2}\right)=\delta\left(K, \operatorname{conv}\left(G_{1} \cup G_{2}\right)\right)$; if $K_{1}, K_{2}$, and $G$ are convex, then $\delta\left(K_{1}, \delta\left(K_{2}, G\right)\right)=\delta\left(\operatorname{conv}\left(K_{1} \cup K_{2}\right), G\right)$.

Lemma 1. Suppose $\mathscr{K}$ is a family of convex sets, $K \in \varphi(\mathscr{K})$, and $G$ is also a convex set. Then

$$
\delta(K, G) \in \varphi(\{\delta(W, G): W \in \mathscr{K}\}) .
$$

Proof. Suppose $T \in \tau(\{\delta(W, G): W \in \mathscr{K}\})$. For each element $W \in \mathscr{K}$, let $p_{W}=$ $a_{W} g_{W}+\left(1-a_{W}\right) x_{W}$ be an element of $T \cap \delta(W, G)$, where $0 \leq a_{W}<1, g_{W} \in G$, and $x_{W} \in W$. Let $T^{\prime}=\operatorname{conv}\left\{x_{W}: W \in \mathscr{K}\right\}$. Clearly, $T^{\prime} \in \tau(\mathscr{K})$, so $T^{\prime} \cap K \neq \varnothing$. Let $w$ be an element of $T^{\prime} \cap K$. Since $w \in T^{\prime}$, we may write $w=\sum_{W \in \mathscr{K}} \beta_{W} x_{W}$, where $\beta_{W}=0$ except for finitely many $W \in \mathscr{K}, \beta_{W} \geq 0$ for $W \in \mathscr{K}$, and $\sum_{W \in \mathscr{K}} \beta_{W}=1$ (so that the sum is a convex combination of the $x_{W}$ 's). Let $\sigma=\sum_{W \in \mathscr{K}}\left[\beta_{W} /\left(1-a_{W}\right)\right]$. Then $\sigma>1$. Let $\gamma_{W}=\beta_{W} /\left[\left(1-a_{W}\right) \sigma\right]$ for $W \in \mathscr{K}$. Then

$$
\begin{aligned}
\sum_{W \in \mathscr{K}} \gamma_{W} p_{W} & =\sum_{W \in \mathscr{K}} \gamma_{W}\left(a_{W} g_{W}+\left(1-a_{W}\right) x_{W}\right) \\
& =\sum_{W \in \mathscr{K}} \frac{\beta_{W} a_{W}}{\left(1-a_{W}\right) \sigma} g_{W}+\sum_{W \in \mathscr{K}} \frac{\beta_{W}}{\sigma} x_{W} \\
& =\frac{\sigma-1}{\sigma}\left(\sum_{W \in \mathscr{K}} \frac{\beta_{W} /\left(1-a_{W}\right)-\beta_{W}}{\sigma-1} g_{W}\right)+\frac{1}{\sigma} w .
\end{aligned}
$$

From the first expression we see that this point is in $T$. From the last we see that it is in $\delta(K, G)$. Then $T \cap \delta(K, G) \neq \varnothing$. Since this is true of each such transversal $T$, it follows that $\delta(K, G) \in \varphi(\{\delta(W, G): W \in \mathscr{K}\})$.

Suppose $\mathscr{K}=\left\{K_{\lambda}: \lambda \in \Lambda\right\}$ is a finite collection of convex sets indexed by $\Lambda$, and suppose $p \in \mathscr{R}^{d}$. Let

$$
\beta(\mathscr{K}, p)=\left\{A \subseteq \Lambda: p \notin \operatorname{conv}\left(\bigcup_{\lambda \in A} K_{\lambda}\right)\right\} .
$$


Lemma 2. Suppose $\mathscr{K}$ is a nonempty collection of convex sets in $\mathscr{R}^{d}$ indexed by $\Lambda$, $a$ and $b$ are distinct point of $\Re^{d}$, and $p$ is a point of the relative interior of the line segment connecting $a$ and $b$. Let

$$
\begin{aligned}
& \mathscr{K}_{1}=\left\{\delta\left(K_{\lambda},\{a\}\right): \lambda \in \Lambda\right\}, \\
& \mathscr{K}_{2}=\left\{\delta\left(K_{\lambda},\{b\}\right): \lambda \in \Lambda\right\},
\end{aligned}
$$

and

$$
\mathscr{K}_{3}=\left\{\delta\left(K_{\lambda}, \operatorname{conv}\{a, b\}\right): \lambda \in \Lambda\right\}
$$

Then

$$
\beta\left(\mathscr{K}_{1}, p\right) \cup \beta\left(\mathscr{K}_{2}, p\right)=\beta(\mathscr{K}, p)
$$

and

$$
\beta\left(\mathscr{K}_{1}, p\right) \cap \beta\left(\mathscr{K}_{2}, p\right)=\beta\left(\mathscr{K}_{3}, p\right)
$$

Proof. We establish the first equality. Suppose $A \in \beta\left(\mathscr{K}_{1}, p\right) \cup \beta\left(\mathscr{K}_{2}, p\right)$. Then

$$
\begin{aligned}
& p \notin \operatorname{conv}\left(\bigcup_{\lambda \in A} \delta\left(K_{\lambda},\{a\}\right)\right) \cap \operatorname{conv}\left(\bigcup_{\lambda \in A} \delta\left(K_{\lambda},\{b\}\right)\right) \\
& =\delta\left(\operatorname{conv}\left(\bigcup_{\lambda \in A} K_{\lambda}\right),\{a\}\right) \cap \delta\left(\operatorname{conv}\left(\bigcup_{\lambda \in A} K_{\lambda}\right),\{b\}\right) \\
& \supseteq \operatorname{conv}\left(\bigcup_{\lambda \in A} K_{\lambda}\right),
\end{aligned}
$$

so $A \in \beta(\mathscr{K}, p)$. Suppose $A \notin \beta\left(\mathscr{K}_{1}, p\right) \cup \beta\left(\mathscr{K}_{2}, p\right)$. Then $p \in \delta\left(\operatorname{conv}\left(\bigcup_{\lambda \in A} K_{\lambda}\right)\right.$, $\{a\}) \cap \delta\left(\operatorname{conv}\left(\bigcup_{\lambda \in A} K_{\lambda}\right),\{b\}\right)$, so there exist $x, y \in \operatorname{conv}\left(\bigcup_{\lambda \in A} K_{\lambda}\right)$ such that $p \in \delta(\{x\},\{a\}) \cap \delta(\{y\},\{b\})$. Since $p$ is strictly between $a$ and $b$ it is clear that $x, y, a, b$, and $p$ are colinear, and that $p \in \operatorname{conv}(\{x, y\})$. Therefore $p \in \operatorname{conv}\left(\bigcup_{\lambda \in A} K_{\lambda}\right)$, so that $A \notin \beta(\mathscr{K}, p)$.

Now we establish the second equality. Suppose $A \in \beta\left(\mathscr{K}_{3}, p\right)$. Then

$$
\begin{aligned}
& p \notin \operatorname{conv}\left(\bigcup_{\lambda \in A} \delta\left(K_{\lambda}, \operatorname{conv}\{a, b\}\right)\right) \\
& \supseteq \operatorname{conv}\left(\bigcup_{\lambda \in A} \delta\left(K_{\lambda},\{a\}\right)\right) \cup \operatorname{conv}\left(\bigcup_{\lambda \in A} \delta\left(K_{\lambda},\{b\}\right)\right)
\end{aligned}
$$

so $A \in \beta\left(\mathscr{K}_{1}, p\right) \cap \beta\left(\mathscr{K}_{2}, p\right)$. Suppose $A \notin \beta\left(\mathscr{K}_{3}, p\right)$. Then

$$
p \in \operatorname{conv}\left(\bigcup_{\lambda \in A} \delta\left(K_{\lambda}, \operatorname{conv}\{a, b\}\right)\right)=\delta\left(\operatorname{conv}\left(\bigcup_{\lambda \in A} K_{\lambda}\right), \operatorname{conv}\{a, b\}\right)
$$


so there is $x \in \operatorname{conv}\left(\bigcup_{\lambda \in A} K_{\lambda}\right)$ such that $p \in \delta(\{x\}$, conv $\{a, b\})$. Since $p$ is on the line through $a$ and $b$, it is clear that $x$ must be, as well. Then

$$
\begin{aligned}
p \in(\operatorname{conv}\{a, b\}) \sim\{a, b\} & \subseteq \delta(\{x\},\{a\}) \cup \delta(\{x\},\{b\}) \\
& \subseteq \delta\left(\operatorname{conv}\left(\bigcup_{\lambda \in A} K_{\lambda}\right),\{a\}\right) \cup \delta\left(\operatorname{conv}\left(\bigcup_{\lambda \in A} K_{\lambda}\right),\{b\}\right) \\
& =\operatorname{conv}\left(\bigcup_{\lambda \in A} \delta\left(K_{\lambda},\{a\}\right)\right) \cup \operatorname{conv}\left(\bigcup_{\lambda \in A} \delta\left(K_{\lambda},\{b\}\right)\right),
\end{aligned}
$$

so $A \notin \beta\left(\mathscr{K}_{1}, p\right) \cap \beta\left(\mathscr{K}_{2}, p\right)$.

If $\mathscr{K}$ is finite, indexed by the finite set $\Lambda=[m]$, then $\beta(\mathscr{K}, p)$ is a finite simplicial complex. Let $\chi$ be the function which assigns to each (finite) simplicial complex its Euler characteristic.

Theorem 3. Let $\mathscr{K}=\left\{K_{i}: i \in[m]\right\}$ and $\mathscr{K}^{\prime}=\left\{K_{j}^{\prime}: j \in[n]\right\}$ be nonempty, finite, indexed collections of convex sets, and let $p$ be a point of $\mathscr{R}^{d}$. Suppose that $\tau(\mathscr{K})=\tau\left(\mathscr{K}^{\prime}\right) \quad\left(\right.$ or, equivalently, that $\left.\varphi(\mathscr{K})=\varphi\left(\mathscr{K}^{\prime}\right)\right)$. Then $\chi(\beta(\mathscr{K}, p))=$ $\chi\left(\beta\left(\mathscr{K}^{\prime}, p\right)\right)$.

Proof. Suppose that this is not the case. Choose $\mathscr{K}, \mathscr{K}^{\prime}$, and $p$ so that the hypotheses are satisfied but $\chi(\beta(\mathscr{K}, p)) \neq \chi\left(\beta\left(\mathscr{K}^{\prime}, p\right)\right)$, in such a way that $|\beta(\mathscr{K}, p)|+\left|\beta\left(\mathscr{K}^{\prime}, p\right)\right|$ is as small as possible.

Clearly, $\quad\{p\} \notin \tau(\mathscr{K} \cup\{\{p\}\}) \quad\left(=\tau\left(\mathscr{K}^{\prime} \cup\{\{p\}\}\right)\right)$, for otherwise $\beta(\mathscr{K}, p)=$ $\beta\left(\mathscr{K}^{\prime}, p\right)=\{\varnothing\}$, and the Euler characteristics would be 0 . Also, it cannot be the case that there are sets $A \subseteq[m]$ and $A^{\prime} \subseteq[n]$ such that

$$
\beta(\mathscr{K}, p)=\{S \subseteq[m]: S \cap A=\varnothing\}
$$

and

$$
\beta\left(\mathscr{K}^{\prime}, p\right)=\left\{T \subseteq[n]: T \cap A^{\prime}=\varnothing\right\}
$$

for then (since we have seen that neither $A=[m]$ nor $A^{\prime}=[n]$ ) the Euler characteristics would both be 1 .

It follows that, for at least one of the two complexes, there is a subset $B$ of the index set which (i) is not in the complex, (ii) has the property that each proper subset is in the complex, and (iii) has cardinality at least 2 . For definiteness, we assume that this is true of the complex $\beta(\mathscr{K}, p)$, so that $B \subseteq[m]$. Let $j$ be an element of $B$. Let $B_{0}=B \sim\{j\}$. We may choose $a \in K_{j}$ and $b \in \operatorname{conv}\left(\bigcup_{i \in B_{0}} K_{i}\right)$ such that $p \in \operatorname{conv}\{a, b\}$. Notice that, since both $\{j\}$ and $B_{0}$ are in $\beta(\mathscr{K}, p)$, $p \notin\{a, b\}$. For $\mathscr{K}, a, b$, and $p$, the situation is that of Lemma 2. Let $\mathscr{K}_{1}, \mathscr{K}_{2}$, and $\mathscr{K}_{3}$ be as in Lemma 2. Also it is clear that Lemma 2 applies to $\mathscr{K}^{\prime}, a, b$, and $p$. Let $\mathscr{K}_{1}^{\prime}, \mathscr{K}_{2}^{\prime}$, and $\mathscr{K}_{3}^{\prime}$ be the corresponding collections in this case.

By Lemma 2 and the modular property of the Euler characteristic,

$$
\chi(\beta(\mathscr{K}, p))=\chi\left(\beta\left(\mathscr{K}_{1}, p\right)\right)+\chi\left(\beta\left(\mathscr{K}_{2}, p\right)\right)-\chi\left(\beta\left(\mathscr{K}_{3}, p\right)\right)
$$


and

$$
\chi\left(\beta\left(\mathscr{K}_{1}^{\prime}, p\right)\right)+\chi\left(\beta\left(\mathscr{K}_{2}^{\prime}, p\right)\right)-\chi\left(\beta\left(\mathscr{K}_{3}^{\prime}, p\right)\right)=\chi\left(\beta\left(\mathscr{K}^{\prime}, p\right)\right) .
$$

The complexes appearing on the right in the first equation are properly contained in $\beta(\mathscr{K}, \boldsymbol{p})$.

Since $\varphi(\mathscr{K})=\varphi\left(\mathscr{K}^{\prime}\right)$, Lemma 1 yields $\varphi\left(\mathscr{K}_{1}\right)=\varphi\left(\mathscr{K}_{1}^{\prime}\right), \varphi\left(\mathscr{K}_{2}\right)=\varphi\left(\mathscr{K}_{2}^{\prime}\right)$, and $\varphi\left(\mathscr{K}_{3}\right)=\varphi\left(\mathscr{K}_{3}^{\prime}\right)$. It follows, considering the minimality criterion for our choice of $\mathscr{K}$ and $\mathscr{K}^{\prime}$, that $\chi\left(\beta(\mathscr{K}, p)=\chi\left(\beta\left(\mathscr{K}^{\prime}, p\right)\right.\right.$, a contradiction.

Recall from [12] that a family of convex sets is clustered if $\tau(\mathscr{K})=$ $\tau\left(\left\{\bigcap_{K \in \mathscr{K}} K\right\}\right)$.

Corollary. If $\mathscr{K}$ is a finite, clustered family of convex sets indexed by $[m]$ then

$$
\chi(\beta(\mathscr{K}, p))= \begin{cases}1 & \text { if } p \in \bigcap_{K \in \mathscr{K}} K, \\ 0 & \text { otherwise. }\end{cases}
$$

\section{Valuations and the Transversal Characteristic}

In this section we utilize facts about valuation modules to produce a partial converse to Theorem 3 .

If $\mathscr{K}$ is a finite family of convex sets in $\mathscr{R}^{d}$ then the function $f: \mathscr{R}^{d} \rightarrow \mathbb{Z}$ given by the formula $f(p)=1-\chi(\beta(\mathscr{K}, p))$ is an element of the additive group of simple functions generated by the characteristic functions of convex sets. Indeed,

$$
f(p)=\sum_{\substack{\mathscr{A} \subseteq \mathscr{K}, \mathscr{A} \neq \varnothing}}(-1)^{|\mathscr{A}|-1} C\left(\operatorname{conv}\left(\bigcup_{K \in \mathscr{A}} K\right), p\right)
$$

where

$$
C(W, p)= \begin{cases}1 & \text { if } p \in W \\ 0 & \text { otherwise }\end{cases}
$$

(We shall usually designate this function simply by $C(W)$, and write $C(W)(p)$ instead of $C(W, p)$.) We denote the function $f$ by $\omega_{\mathscr{K}}$ or $\omega(\mathscr{K})$, and call it the transversal characteristic of $\mathscr{K}$.

Suppose $\mathscr{D}$ is a distributive lattice of subsets of $\mathscr{R}^{d}$, and $\varnothing \in \mathscr{D}$. Let $S(\mathscr{D})$ denote the group of simple functions generated by the characteristic functions of elements of $\mathscr{D}$. Let $C: \mathscr{D} \rightarrow S(\mathscr{D})$ be the mapping which takes $D \in \mathscr{D}$ to its characteristic function. Then each valuation $v$ on $\mathscr{D}$, having values in an abelian group $A$, such that $v(\varnothing)=0$, induces a homorphism $\bar{v}: S(\mathscr{D}) \rightarrow A$, the unique homomorphism such that $v(D)=\bar{v}(C(D)$ ) for $D \in \mathscr{D}$. (See [2, I and III].) Obviously, given any homomorphism $\bar{v}: S(\mathscr{D}) \rightarrow A$, the composition $\bar{v} \circ C$ is a valuation on $\mathscr{D}$. 
Theorem 4. Suppose $\mathscr{K}$ and $\mathscr{K}^{\prime}$ are finite, nonempty families of convex sets and that $\tau(\mathscr{K})=\tau\left(\mathscr{K}^{\prime}\right)$. Suppose that $\mathscr{D}$ is a distributive lattice of sets which contains $\operatorname{conv}\left(\bigcup_{K \in \mathscr{A}} K\right)$, for each subset $\mathscr{A}$ of $\mathscr{K} \cup \mathscr{K}^{\prime}$. If $v: \mathscr{D} \rightarrow A$ is a valuation on $\mathscr{D}$, then

$$
\sum_{\substack{\mathscr{A} \subseteq \mathscr{K}, \mathscr{A} \neq \varnothing}}(-1)^{|\mathscr{A}|-1} v\left(\operatorname{conv}\left(\bigcup_{K \in \mathscr{A}} K\right)\right)=\sum_{\substack{\mathscr{A} \subseteq \mathscr{T}^{\prime} \\ \mathscr{A} \neq \varnothing}}(-1)^{|\mathscr{A}|-1} v\left(\operatorname{conv}\left(\bigcup_{K \in \mathscr{A}} K\right)\right) .
$$

Proof. Suppose, first, that $v(\varnothing)=0$. Let $\bar{v}$ be the corresponding group homomorphism, $\bar{v}: S(\mathscr{D}) \rightarrow A$. If $\tau(\mathscr{K})=\tau\left(\mathscr{K}^{\prime}\right)$ then, by Theorem $3, \omega(\mathscr{K})=\omega\left(\mathscr{K}^{\prime}\right)$, so that $\bar{v}(\omega(\mathscr{K}))=\bar{v}\left(\omega\left(\mathscr{K}^{\prime}\right)\right)$. This immediately yields the desired equality.

The result now follows in general by noting that (i) any valuation is the sum of a constant valuation and one which maps $\varnothing$ to zero, and (ii) the result holds for constant valuations.

Corollary. Suppose $\mathscr{K}$ is a finite, clustered family of convex sets, and that $\mathscr{D}$ is a distributive lattice of sets such that $\operatorname{conv}\left(\bigcup_{K \in \mathscr{A}} K\right) \in \mathscr{D}$, for each collection $\mathscr{A} \subseteq \mathscr{K}$. Let $v: \mathscr{D} \rightarrow A$ be a valuation on $\mathscr{D}$. Then

$$
\sum_{\substack{\mathscr{A} \subseteq \mathscr{K}, \mathscr{A} \neq \varnothing}}(-1)^{|\cdot \mathscr{A}|-1} v\left(\operatorname{conv}\left(\bigcup_{K \in \mathscr{A}} K\right)\right)=v\left(\bigcap_{K \in \mathscr{K}} K\right)
$$

Proof. This follows at once from Theorem 4 by setting $\mathscr{K}^{\prime}=\left\{\bigcap_{K \in \mathscr{K}} K\right\}$.

Next we have, essentially, the converse to Theorem 3, when the sets are open.

Theorem 5. Suppose $\mathscr{K}$ and $\mathscr{K}^{\prime}$ are finite, nonempty families of open, convex sets in $\mathscr{R}^{d}$. Then $\tau(\mathscr{K})=\tau\left(\mathscr{K}^{\prime}\right)$ if and only if $\omega(\mathscr{K})=\omega\left(\mathscr{K}^{\prime}\right)$.

Proof. If $\tau(\mathscr{K})=\tau\left(\mathscr{K}^{\prime}\right)$ then $\omega(\mathscr{K})=\omega\left(\mathscr{K}^{\prime}\right)$, by Theorem 3 .

Suppose $\omega(\mathscr{K})=\omega\left(\mathscr{K}^{\prime}\right)$. Let $\chi_{\mathrm{o}}$ be the Euler characteristic for the lattice of finite unions of open, convex sets in $\mathscr{R}^{d}$. Let $T$ be an element of $\tau(\mathscr{K})$. If $T \notin \tau\left(\mathscr{K}^{\prime}\right)$ then there is an element $K \in \mathscr{K}^{\prime}$ and a closed half-space $H$ such that $T \subseteq H$ and $K \cap, H=\varnothing$. Let $H^{\prime}$ be the interior of $H$. The function $v(G)=\chi_{0}\left(G \cap H^{\prime}\right)$ is a valuation on the lattice of finite unions of open convex sets. It induces a homomorphism, $\bar{v}: S_{\mathrm{o}} \rightarrow \mathbb{Z}$, where $S_{\mathrm{o}}$ is the group generated by the characteristic functions of open convex sets.

Since $H \in \tau(\mathscr{K}), H=\mathrm{cl} H^{\prime}$, and all elements of $\mathscr{K}$ are open, it follows that $H^{\prime} \in \tau(\mathscr{K})$. Then for each nonempty subset $\mathscr{A}$ of $\mathscr{K}$ it is clear that (conv $\left.\left(\bigcup_{A \in \mathscr{A}} A\right) \cap H^{\prime}\right)$ is a nonempty, open, convex set, so its Euler characteristic is 1 . It follows that $\bar{v}(\omega(\mathscr{K}))=1$.

Let $\mathscr{B}=\left\{U \in \mathscr{K}^{\prime}: U \cap H^{\prime}=\varnothing\right\}$. Clearly, $K \in \mathscr{B}$, so $\mathscr{B} \neq \varnothing$. For nonempty $\mathscr{A} \subseteq$ $\mathscr{K}^{\prime},\left(\operatorname{conv}\left(\bigcup_{U \in \mathscr{A}} U\right) \cap H^{\prime}\right)$ is an open, convex set which is empty if and only if $\mathscr{A} \subseteq \mathscr{B}$. It follows that $\bar{v}\left(\omega\left(\mathscr{K}^{\prime}\right)\right)=0$. This is a contradiction, since $\omega(\mathscr{K})=\omega\left(\mathscr{K}^{\prime}\right)$; so it must be the case that $T \in \tau\left(\mathscr{K}^{\prime}\right)$.

We have shown that $\tau(\mathscr{K}) \subseteq \tau\left(\mathscr{K}^{\prime}\right)$. The reverse inclusion becomes apparent upon reversing the roles of $\mathscr{K}$ and $\mathscr{K}^{\prime}$ in the foregoing. 
In Section 6 we consider another converse to Theorem 3, when the sets involved are assumed to be closed.

\section{The Open, Polar Lattice}

Let $\mathscr{A}$ be an additive, abelian group. Let $S$ be a set endowed with a binary operation, $\Lambda$, under which it is a semilattice. Suppose, further, that there is an injection $\varepsilon: S \rightarrow \mathscr{A}$. For $T \subseteq S$ of finite cardinality and nonempty, let

$$
\mu(T)=\sum_{\substack{U \subseteq T \\ U \neq \varnothing}}(-1)^{|U|-1} \varepsilon\left(\bigwedge_{u \in U} u\right)
$$

We say that the semigroup $(S, \wedge)$ is conforming (relative to $\varepsilon$ ) if, given any finite sets $T_{1}, T_{1}^{\prime}, T_{2}$, and $T_{2}^{\prime}$ contained in $S$ such that $\mu\left(T_{1}\right)=\mu\left(T_{1}^{\prime}\right)$ and $\mu\left(T_{2}\right)=\mu\left(T_{2}^{\prime}\right)$, one has $\mu\left(T_{1} \cup T_{2}\right)=\mu\left(T_{1}^{\prime} \cup T_{2}^{\prime}\right)$.

Suppose $(S, \wedge)$ is conforming. Let $L$ denote the image of $\mu$, so that $L=\{\mu(T)$ : $T \subseteq S, 0<|T|<\infty\} \subseteq \mathscr{A}$. We define a binary operation $\vee$ on $L$ by the rule $a \vee b=\mu\left(T_{1} \cup T_{2}\right)$, where $a=\mu\left(T_{1}\right)$ and $b=\mu\left(T_{2}\right)$. This is well defined, since $(S, \wedge)$ is conforming. For $a, b \in L$ we define $a \wedge b=a+b-a \vee b$. Then, for $s, t \in S$, one has $\varepsilon(s \wedge t)=\varepsilon(s) \wedge \varepsilon(t)$, since by definition $\varepsilon(s) \vee \varepsilon(t)=\mu(\{s, t\})=$ $\varepsilon(s)+\varepsilon(t)-\varepsilon(s \wedge t)$. We use this formula to extend this operation to $L$. Presently, we shall see that if $a$ and $b$ are in $L$ then $a \wedge b$ is, as well. We shall call the operations $\wedge$ and $\vee$ "meet" and "join".

Theorem 6. If $(S, \wedge)$ is a conforming semilattice, and $L, \wedge$, and $\vee$ are as above, then $(L, \wedge, \vee)$ is a distributive lattice. The inclusion $c: L \rightarrow \mathscr{A}$ is a valuation on $L$.

Proof. The operation $\vee$ on $L$ is clearly commutative, associative, and idempotent, so $(L, \vee)$ is a semilattice. It is easily verified that

$$
\varepsilon\left(a_{1}\right) \vee \cdots \vee \varepsilon\left(a_{k}\right)=\mu\left(\left\{a_{1}, \ldots, a_{k}\right\}\right) \quad \text { for } \quad a_{1}, \ldots, a_{k} \in S
$$

In particular, $L$ is generated under $\vee$ by the image, $\varepsilon(S)$, of $S$.

Suppose $a, b_{1}, \ldots, b_{k}$ are in $\varepsilon(S)$. Then

$$
\begin{aligned}
a \wedge\left(b_{1} \vee \cdots \vee b_{k}\right) & =a+\left(b_{1} \vee \cdots \vee b_{k}\right)-\left(a \vee b_{1} \vee \cdots \vee b_{k}\right) \\
& =-\sum_{1 \leq i_{1} \leq \vee \leq i_{1} \leq k}(-1)^{r}\left(a \wedge b_{i_{1}} \wedge \cdots \wedge b_{i_{r}}\right) \\
& =\left(a \wedge b_{1}\right) \vee \cdots \vee\left(a \wedge b_{k}\right) .
\end{aligned}
$$

It follows that

$$
\left(a_{1} \vee \cdots \vee a_{k}\right) \wedge\left(b_{1} \vee \cdots \vee b_{1}\right)=\bigvee_{\substack{i \in[k], j \in[]]}}\left(a_{i} \wedge b_{j}\right)
$$


whenever $a_{i}, b_{j} \in \varepsilon(S)(i \in[k], j \in[l])$. Since, as we have seen, $L$ is precisely the set of joins of elements of $\varepsilon(S), L$ is closed under $\wedge$.

It is not difficult to verify by using $(*)$ that $\wedge$ is associative, commutative, and idempotent, and that $\wedge$ distributes over $\vee$.

The identity $a \wedge b=a+b-(a \vee b)$ establishes the final statement of the theorem.

For example, suppose $\mathscr{A}$ is the abelian group generated, under addition, by the characteristic functions of sets in a family $S$ which is closed under finite intersection. There is the obvious injection $\varepsilon: S \rightarrow \mathscr{A}$, sending each element of $S$ to its characteristic function. If $\wedge$ denotes intersection (making $S$ into a semilattice), then $S$ is conforming. The lattice $L$ is isomorphic to the lattice of finite unions of elements of $S$.

Let $S_{\mathrm{o}}$ denote the abelian group generated by the characteristic functions of open, convex sets in $\mathscr{R}^{d}$. We utilize the mapping taking each convex, open set to its characteristic function.

Theorem 7. The semilattice of open, convex sets in $\mathscr{R}^{d}$ under the operation $U \wedge V=$ conv $(U \cup V)$ is conforming in $S_{\mathrm{o}}$.

Proof. In this case, the function $\mu$ described above coincides with the transversal characteristic, $\omega$.

By Theorem 5 , if $\mathscr{K}$ and $\mathscr{K}^{\prime}$ are finite, nonempty families of open, convex sets such that $\omega(\mathscr{K})=\omega\left(\mathscr{K}^{\prime}\right)$, then $\tau(\mathscr{K})=\tau\left(\mathscr{K}^{\prime}\right)$. If $\omega\left(\mathscr{K}_{1}\right)=\omega\left(\mathscr{K}_{1}^{\prime}\right)$ and $\omega\left(\mathscr{K}_{2}\right)=\omega\left(\mathscr{K}_{2}^{\prime}\right)$ then $\tau\left(\mathscr{K}_{1}\right)=\tau\left(\mathscr{K}_{1}^{\prime}\right)$ and $\tau\left(\mathscr{K}_{2}\right)=\tau\left(\mathscr{K}_{2}^{\prime}\right)$. From these equalities follows

$$
\begin{aligned}
\tau\left(\mathscr{K}_{1} \cup \mathscr{K}_{2}\right) & =\tau\left(\mathscr{K}_{1}\right) \cap \tau\left(\mathscr{K}_{2}\right) \\
& =\tau\left(\mathscr{K}_{1}^{\prime}\right) \cap \tau\left(\mathscr{K}_{2}^{\prime}\right) \\
& =\tau\left(\mathscr{K}_{1}^{\prime} \cup \mathscr{K}_{2}^{\prime}\right),
\end{aligned}
$$

so that $\omega\left(\mathscr{K}_{1} \cup \mathscr{K}_{2}\right)=\omega\left(\mathscr{K}_{1}^{\prime} \cup \mathscr{K}_{2}^{\prime}\right)$, as required.

We denote by $\hat{L}_{\mathrm{o}}$ the distributive lattice corresponding to $L$, of Theorem 7 . We call it the polar lattice generated by the open, convex sets, or, briefly, the open, polar lattice. The extension of the convex hull operation is the meet operation on $\hat{L}_{\mathrm{o}}$. We shall, therefore, denote the meet of elements $A$ and $B$ by $A$ conv $B$.

Lemma 3. The zero function is the maximal element of $\hat{L}_{o}$. It is irreducible.

Proof. Suppose $Y \in \hat{L}_{\mathrm{o}}$. We must show that $C(\varnothing)$ conv $Y=Y$. (Here, $C(\varnothing)$ denotes the characteristic function of $\varnothing$-the zero function. This function is certainly an element of $\hat{L}_{\mathrm{o}}$, since $C(\varnothing)=\omega(\{\varnothing\})$.) Since $Y \in \hat{L}_{\mathrm{o}}$, there is a family $\mathscr{K}$ of open, convex sets such that $Y=\omega(\mathscr{K})$. Then $C(\varnothing)$ conv $Y=$ $C(\varnothing)+Y-\omega(\mathscr{K} \cup\{\varnothing\})=Y$. It follows that $C(\varnothing)$ is the maximal element of $\hat{L}_{\mathrm{o}}$. 
Suppose $X_{1}=\omega\left(\mathscr{K}_{1}\right)$ and $X_{2}=\omega\left(\mathscr{K}_{2}\right)$. Then the join of $X_{1}$ and $X_{2}$ in $\hat{L}_{\mathrm{o}}$ is $\omega\left(\mathscr{K}_{1} \cup \mathscr{K}_{2}\right)$. It remains only to show that if $\mathscr{K}$ is any finite, nonempty collection of open, nonempty convex sets then $\omega(\mathscr{K})$ is not identically 0 . Let $\bar{\chi}_{0}$ denote the homomorphism, $\bar{\chi}_{\mathrm{o}}: S_{\mathrm{o}} \rightarrow \mathbb{Z}$, induced by the Euler characteristic. Then we have $\bar{X}_{\mathrm{o}}(\omega(\mathscr{K}))=1$, under the stated conditions on $\mathscr{K}$. It follows that $\omega(\mathscr{K})$ is not the zero function.

We denote by $\hat{L}_{\mathrm{o}}^{\prime}$ the lattice $\hat{L}_{o}$ with the irreducible, maximal element, $C(\varnothing)$, removed. It follows from Theorems 6 and 7 that the natural injection $\hat{L}_{\mathrm{o}} \rightarrow S_{\mathrm{o}}$, as well as its restriction to $\hat{L}_{0}^{\prime}$, is a valuation. It follows that it extends to a homomorphism, $\eta: V\left(\hat{L}_{o}^{\prime}\right) \rightarrow S_{o}$, where $V\left(\hat{L}_{o}^{\prime}\right)$ denotes the valuation ring of $\hat{L}_{o}^{\prime}$. Our next objective is to show that this mapping is an isomorphism. First, we develop a useful criterion for determining if an element $X \in S_{\mathrm{o}}$ is the zero function.

By an elementary valuation on a distributive lattice $L$, we mean a valuation $e: L \rightarrow \mathbb{Z}$, whose image is $\{0,1\}$, such that if $a, b \in L$ and $a \leq b$, then $e(a) \leq e(b)$. Given a valuation $v: L \rightarrow \mathbb{Z}$, let $\tilde{v}$ denote its unique extension to a homomorphism, $\tilde{v}: V(L) \rightarrow \mathbb{Z}$.

Lemma 4. Let $L$ be a distributive lattice. Let $W$ be a set of elementary valuations on $L$ which distinguish points of $L$. Let $e_{0}$ be the valuation which is identically 1 on $L$. Finally, let $\tilde{W}=\{\tilde{e}: e \in W\}$. Then the elements of $\tilde{W} \cup\left\{\tilde{e}_{a}\right\}$ distinguish points of $V(L)$. If $L$ has no least element, then the elements of $\tilde{W}$ distinguish points of $V(L)$.

Proof. For $x \in L$, let $\delta(x)=\left\{e \in W \cup\left\{e_{0}\right\} ; e(x)=1\right\}$. Then $\delta$ is a lattice monomorphism of $L$ into the lattice of subsets of $W \cup\left\{e_{0}\right\}$. Let $\delta(L)$ denote its image, so that $L \simeq \delta(L)$. Notice that $e_{0} \in \delta(x)$, for each $x$ in $L$. Then $\delta(L)$ is a lattice of nonempty sets. (Notice that, if $L$ has no least element, then $\delta(x) \sim\left\{e_{0}\right\}$ is nonempty.) Let $S$ denote the group of simple functions on $W$ generated (under addition) by the characteristic functions of sets $\delta(x)$. According to [2, III], the unique extension to $V(\delta(L))$ of the valuation on $\delta(L)$ taking $\delta(x)$ to its characteristic function is an isomorphism. Composing, we have a valuation $\psi: L \rightarrow S$ whose extension $\tilde{\psi}: V(L) \rightarrow S$ is an isomorphism. Suppose $v_{1}$ and $v_{2}$ are distinct elements of $V(L)$. Then $\tilde{\psi}\left(v_{1}-v_{2}\right) \neq 0$, and we may choose $e \in W$ on which it is nonzero; but its value on $e$ is, clearly, $\tilde{e}\left(v_{1}\right)-\tilde{e}\left(v_{2}\right)$, so $\tilde{e}$ distinguishes $v_{1}$ and $v_{2}$.

The final statement in the lemma is clear, considering the parenthetical comment above.

Let $H$ be an open half-space. Then the function $K \mapsto \chi_{\mathrm{o}}(K \cap H)$ is a valuation on $L_{\mathrm{o}}$, the lattice of finite unions of open, convex sets. Its value on the empty set is 0 , so it extends uniquely to a homomorphism $\bar{v}_{H}: S_{\mathrm{o}} \rightarrow \mathbb{Z}$. Also, let $\bar{\chi}_{\mathrm{o}}: S_{\mathrm{o}} \rightarrow \mathbb{Z}$ be the extension of $\chi_{0}$ to $S_{0}$.

Theorem 8. The function $\eta: V\left(\hat{L}_{\mathrm{o}}^{\prime}\right) \rightarrow S_{\mathrm{o}}$ is an isomorphism.

Proof. If $H$ is an open half-space, let $v_{H}^{\prime}(F)=1-\bar{v}_{H}(F)$, for $F \in \hat{L}_{\mathrm{o}}$. We show, first, that the restriction of $v_{\mathrm{H}}^{\prime}$ to $\hat{L}_{\mathrm{o}}$ is an elementary valuation. Clearly, it is a 
valuation. Suppose $F=\omega(\mathscr{K})$, where $\mathscr{K}$ is a finite, nonempty collection of open, convex sets. Then

$$
v_{H}^{\prime}(F)= \begin{cases}1 & \text { if } H \notin \tau(\mathscr{K}), \\ 0 & \text { if } H \in \tau(\mathscr{K}) .\end{cases}
$$

It is clear from this that if $\mathscr{K}_{1} \subseteq \mathscr{K}_{2}$ then $v_{H}^{\prime}\left(\omega\left(\mathscr{K}_{1}\right)\right) \leq v_{H}^{\prime}\left(\omega\left(\mathscr{K}_{2}\right)\right)$, which implies that $v_{H}^{\prime}$ is monotone on $\hat{L}_{\mathrm{o}}$.

Next, we establish that elements of $\hat{L}_{\mathrm{o}}$ are distinguished by valuations of the form $v_{H}^{\prime}$. Suppose that $F_{1}=\omega\left(\mathscr{K}_{1}\right)$ and $F_{2}=\omega\left(\mathscr{K}_{2}\right)$ are elements of $\hat{L}_{\mathrm{o}}$, and that $F_{1} \neq F_{2}$. Then $\tau\left(\mathscr{K}_{1}\right) \neq \tau\left(\mathscr{K}_{2}\right)$. Suppose, for instance, that $\tau\left(\mathscr{K}_{1}\right) \notin \tau\left(\mathscr{K}_{2}\right)$. Let $T$ be an element of $\tau\left(\mathscr{K}_{1}\right) \sim \tau\left(\mathscr{K}_{2}\right)$. Let $K$ be an element of $\mathscr{K}_{2}$ such that $T \cap K=\varnothing$. There is a closed half-space $H^{\prime}$ such that $T \subseteq H^{\prime}$ and $K \cap H^{\prime}=\varnothing$. Let $H=$ int $H^{\prime}$. Since $T \subseteq H^{\prime}, H^{\prime} \in \tau\left(\mathscr{K}_{1}\right)$. Since $H^{\prime}=\mathrm{cl} H$ and all the elements of $\mathscr{K}_{1}$ are open, $H \in \tau\left(\mathscr{K}_{1}\right)$. However, $H \notin \tau\left(\mathscr{K}_{2}\right)$, since $K \cap H=\varnothing$. It follows that $v_{H}^{\prime}\left(F_{1}\right)=0 \neq 1=$ $v_{H}^{\prime}\left(F_{2}\right)$.

The lemma now yields that the extensions $\tilde{v}_{H}^{\prime}$ to $V\left(\hat{L}_{\mathrm{o}}\right)$ of the functions $v_{H}^{\prime}$, together with that of the function identically 1 on $\hat{L}_{\mathrm{o}}$, distinguish elements of $V\left(\hat{L}_{\mathrm{o}}\right)$. Let the extension of the function identically 1 on $\hat{L}_{\mathrm{o}}$ be $\hat{v}_{\mathrm{o}}: V\left(\hat{L}_{\mathrm{o}}\right) \rightarrow \mathbb{Z}$.

Consider the homomorphism $\eta: V\left(\hat{L}_{\mathrm{o}}^{\prime}\right) \rightarrow S_{\mathrm{o}}$. Clearly, it is surjective. We need only establish that its kernel is $\{0\}$. Suppose $x \in V\left(\hat{L}_{\mathrm{o}}^{\prime}\right)$ and $x \neq 0$. If there is an open half-space $H$ such that $\tilde{v}_{H}^{\prime}(x) \neq 0$ then we have that $0 \neq \tilde{v}_{H}^{\prime}(x)=$ $\bar{\chi}_{0}(\eta(x))-\bar{v}_{H}(\eta(x))$. Clearly, we must have $\eta(x) \neq 0$. Likewise, if $\tilde{v}_{0}(x) \neq 0$, then we have $0 \neq \tilde{v}_{\mathrm{o}}(x)=\bar{\chi}_{\mathrm{o}}(\eta(x))$. (This equality holds in $V\left(\hat{L}_{\mathrm{o}}^{\prime}\right)$ since it holds for elements of $\hat{L}_{\mathrm{o}}^{\prime}$.) Again it follows that $\eta(x) \neq 0$.

We observe the following useful by-product of the proof of Theorem 8 .

Theorem 9. Let $F$ be an element of $S_{0}$. If $F \neq 0$ then there is an open half-space, $H$, such that $\bar{v}_{H}(F) \neq 0$.

Proof. It is clear from the proof of Theorem 8 that the functions $\bar{v}_{H}$ are elementary valuations on the dual of $\hat{L}_{\mathrm{o}}^{\prime}$. This lattice has no least element, so, according to the lemma, they distinguish points of $S_{0}$.

Theorem 10. The binary operation conv on $\hat{L}_{\mathrm{o}}^{\prime}$ has a unique extension to a binary operation on $S_{\mathrm{o}}$ which distributes over addition. With this multiplication, $S_{\mathrm{o}}$ becomes a ring.

Proof. Since $S_{\mathrm{o}}$ is isomorphic to the valuation module of $\hat{L}_{\mathrm{o}}^{\prime}$ and conv is the meet operation of $\hat{L}_{o}^{\prime}$, the multiplication of the valuation ring yields such an extension. Uniqueness is not hard to verify.

Reference [4] contains a result analogous to Theorem 10, but with conv replaced by Minkowski addition. See also [13]. 


\section{The Sallee-Shephard Mapping and the Closed, Polar Lattice}

In this section we will describe a lattice. $\hat{L}_{\mathrm{c}}$, related to the closed, convex sets in roughly the same way that $\hat{L}_{\mathrm{o}}$ is related to the open, convex sets. We discuss its valuation module and show, in fact, that $V\left(\hat{L}_{\mathrm{c}}\right)$ is isomorphic to $V\left(\hat{L}_{\mathrm{o}}\right)$. The isomorphism is what we call the "Sallee-Shephard mapping." Sallee and Shephard studied a function which is essentially an adjoint of this mapping. (See [17] and [18], and, in particular, Theorem 4.2 of [17].)

The section closes with another application of the Sallee-Shephard mapping. We use it to characterize those finite families $\mathscr{P}$ and 2 of convex polytopes which are dually related in the lattice $\Phi_{p}$ of Section 1; i.e., for which $\tau(\mathscr{P})=\varphi(\mathscr{Q})$.

To begin, we describe a homomorphism cl: $S_{\mathrm{o}} \rightarrow S_{\mathrm{c}}$, where $S_{\mathrm{c}}$ is the group of simple functions generated by the closed, convex sets. Given the characteristic function $F=C(U)$ of an open convex set $U$, let cl $F$ denote the characteristic function of its closure. (If $W$ is a set, we also denote its closure by cl $W$. Hopefully, this will not cause confusion.)

Lemma 5. The function $\mathrm{cl}$ extends uniquely to a homomorphism $\mathrm{cl}: S_{\mathrm{o}} \rightarrow S_{\mathrm{c}}$.

Proof. Recall that $\bar{\chi}_{\mathrm{o}}: S_{\mathrm{o}} \rightarrow \mathbb{Z}$ is the homomorphism induced by the Euler characteristic $\chi_{0}: L_{0} \rightarrow \mathbb{Z}$. The desired homomorphism maps $F \in S_{\mathrm{o}}$ to the function whose value at $x$ is $\bar{\chi}_{\mathrm{o}}(F \cap C(B))$, where $B$ is the characteristic function of a sufficiently small, open ball centered at $x$. (Here, $F \cap C(B)$ denotes pointwise multiplication of the functions. The reader will recognize this as the multiplication in an isomorphic copy of the augmentation ideal of the valuation ring of the lattice $\hat{L}_{\mathrm{o}}$.)

To be more precise, we let $F=\sum_{1 \in[n]} \alpha_{i} F_{i}$, where the functions $F_{i}$ are characteristic functions of open, convex sets, $U_{i}$. If $B$ is the characteristic function of any open ball centered at $x$ so small that it does not meet any of the sets $U_{i}$ for which $x \notin \mathrm{cl} U_{i}$, then $\mathrm{cl} F$ has the value

$$
\bar{\chi}_{\circ}(F \cap B)=\sum_{\substack{i \in[n], x \in \operatorname{cl} U_{i}}} \alpha_{i} .
$$

This function is the characteristic function of $\mathrm{cl} U$, if $F$ is the characteristic function of $U$. It is well defined: it is clear from the left-hand side of the above equation that the number does not depend on the decomposition of the function $F$, and it is clear from the right-hand side that it does not depend on which small ball is chosen.

Uniqueness is clear.

Next, we show that $\mathrm{cl}$ is, in fact, an isomorphism. We shall make use of $\chi_{\mathrm{c}}$, the Euler characteristic on the lattice $L_{\mathrm{c}}$, or, rather, of the induced homomorphism $\bar{\chi}_{\mathrm{c}}: S_{\mathrm{c}} \rightarrow \mathbb{Z}$.

Theorem 11. The homomorphism cl: $S_{\mathrm{o}} \rightarrow S_{\mathrm{c}}$ is an isomorphism.

Proof. First we verify that $\mathrm{cl}$ is monomorphic. Suppose $F \in S_{\mathrm{o}}$ and $F \neq 0$. By Theorem 9 there is an open half-space, $H$, such that $\bar{\chi}_{\mathrm{o}}(C(H) \cap F) \neq 0$. We may 
suppose $F=\sum_{i \in\{n]} \alpha_{i} F_{i}$, where $F_{i}$ is the characteristic function of the open, convex set $U_{i}$ (for $i \in[n]$ ). Let $H^{\prime}$ be a closed half-space contained in $H$ such that $U_{i} \cap H^{\prime} \neq \varnothing$ for each $i$ for which $U_{i} \cap H \neq \varnothing$. Then

$$
\begin{gathered}
0 \neq \bar{\chi}_{\mathrm{o}}(C(H) \cap F)=\sum_{i \in[n]} \alpha_{i} \bar{\chi}_{\mathrm{o}}\left(C(H) \cap F_{i}\right) \\
=\sum_{i \in[n]} \alpha_{i} \bar{\chi}_{\mathrm{c}}\left(C\left(H^{\prime}\right) \cap \operatorname{cl}\left(F_{i}\right)\right)=\bar{\chi}_{\mathrm{c}}(\mathrm{cl} F)
\end{gathered}
$$

Therefore $\mathrm{cl} F \neq 0$.

It remains to show that $\mathrm{cl}$ is surjective. For this it is clear that it suffices to show that the characteristic functions of closed, convex sets are in the image. Let $K$ be such a set. If $K$ is $d$-dimensional then $C(K)=\mathrm{cl} F$, where $F$ is the characteristic function of its interior. We may proceed by induction on the codimension of $K$. Suppose $K$ is lower-dimensional, and that the characteristic functions of all closed sets of dimension one more are in the image. It is clear that we may write $K$ as the intersection, $K=K_{1} \cap K_{2}$, of two closed, convex sets of dimension one more than that of $K$, and such that $K_{1} \cup K_{2}$ is also convex. Since $C(K)=C\left(K_{1}\right)+C\left(K_{2}\right)-C\left(K_{1} \cup K_{2}\right)$, it follows that $C(K)$ is also in the image.

Let $\mathrm{cl}^{-1}: S_{\mathrm{c}} \rightarrow S_{\mathrm{o}}$ denote the inverse of cl. We call the function $\sigma=(-1)^{d} \mathrm{cl}^{-1}$ the Sallee-Shephard mapping.

For elements $F$ and $G$ of $S_{\mathrm{c}}$ we define $F$ cl conv $G=\mathrm{cl}\left(\mathrm{cl}^{-1}(F)\right.$ conv cl${ }^{-1}(G)$ ), so that cl conv is a multiplication on $S_{\mathrm{c}}$. Note that if $F$ and $G$ are $d$-dimensional, closed, convex sets then this is the closure of the convex hull of $F$ and $G$. We define $\hat{L}_{\mathrm{c}}$ to be the image of $\hat{L}_{\mathrm{o}}$ under cl, a lattice with operations induced from $\hat{L}_{\mathrm{o}}$. We call it the polar lattice generated by the closed, convex sets. If we let $\hat{L}_{\mathrm{c}}^{\prime}=\hat{L}_{\mathrm{c}} \sim\{C(\varnothing)\}$, then it is clear that $V\left(\hat{L}_{\mathrm{c}}^{\prime}\right) \simeq S_{\mathrm{c}}$.

An easy argument (used, already, in the proof of Theorem 11, and to be used again in that of Theorem 13) shows that $\hat{L}_{\mathrm{c}}$ contains the characteristic functions of all closed, convex sets, and that, if $K$ is such a set, then

$$
\mathrm{cl}^{-1}(C(K))=(-1)^{d-\operatorname{dim} K} C(\text { relint } K) \text {. }
$$

We include another useful theorem.

Theorem 12. Let $F$ be a nonzero element of $S_{\mathrm{c}}$. Then there is a closed half-space $H$ such that $\bar{\chi}_{\mathrm{c}}(C(H) \cap F) \neq 0$.

Proof. Since $F \neq 0, G=\mathrm{cl}^{-1}(F) \neq 0$. Then, by Theorem 9, there is an open half-space, $H^{\prime}$, such that $\bar{\chi}_{\mathrm{o}}\left(C\left(H^{\prime}\right) \cap G\right) \neq 0$. The result follows, with $H=\mathrm{cl} H^{\prime}$.

Next we see that $F \mathrm{cl}$ conv $G$ is what it should be, whenever $F$ and $G$ are characteristic functions of closed, convex sets. 
Theorem 13. If $K_{1}$ and $K_{2}$ are closed, convex sets then $C\left(K_{1}\right) \mathrm{cl}$ conv $C\left(K_{2}\right)=$ $C\left(\operatorname{cl} \operatorname{conv}\left(K_{1} \cup K_{2}\right)\right)$.

Proof. Suppose not. Choose $K_{1}$ and $K_{2}$ for which the identity fails, in such a way that $\operatorname{dim} K_{1}+\operatorname{dim} K_{2}$ is as large as possible, and so that $\operatorname{dim} K_{1} \leq \operatorname{dim} K_{2}$. Then $\operatorname{dim} K_{1} \leq d$; we have already observed that the identity holds when both $K_{1}$ and $K_{2}$ are of dimension $d$, so $\operatorname{dim} K_{1} \leq d-1$. We may write $K_{1}=W_{1} \cap W_{2}$, where $W_{1}, W_{2}$, and $W_{1} \cup W_{2}$ are closed, convex, and of dimension one more than that of $K_{1}$.

Since $C\left(K_{1}\right)$ cl conv $C\left(K_{2}\right) \neq C\left(\operatorname{cl} \operatorname{conv}\left(K_{1} \cup K_{2}\right)\right)$ there is $x \in \mathscr{R}^{d}$ on which these functions differ. Suppose $x \in \mathrm{cl} \operatorname{conv}\left(K_{1} \cup K_{2}\right)$, so that the right-hand side has value 1 . The left-hand side is

$$
\begin{aligned}
C\left(W_{1}\right) \operatorname{cl} \operatorname{conv} C\left(K_{2}\right)+C\left(W_{2}\right) \operatorname{cl} \operatorname{conv} C\left(K_{2}\right) \\
\quad-C\left(W_{1}\right) \operatorname{cl} \operatorname{conv} C\left(W_{2}\right) \operatorname{cl} \operatorname{conv} C\left(K_{2}\right) \\
=C\left(\operatorname{cl} \operatorname{conv}\left(W_{1} \cup K_{2}\right)\right)+C\left(\operatorname{cl} \operatorname{conv}\left(W_{2} \cup K_{2}\right)\right) \\
\quad-C\left(\operatorname{cl} \operatorname{conv}\left(W_{1} \cup W_{2} \cup K_{2}\right)\right) .
\end{aligned}
$$

Since $W_{1}$ and $W_{2}$ contain $K_{1}$, this expression has value 1 on $x$.

We may now suppose that $x \notin \operatorname{cl} \operatorname{conv}\left(K_{1} \cup K_{2}\right)$, so that, at $x$, the right-hand side has value 0 . Then there is a closed half-space $H$ such that $x \in H$ and $H \cap \operatorname{cl} \operatorname{conv}\left(K_{1} \cup K_{2}\right)=\varnothing$. Since $H \cap W_{1} \cap W_{2}=\varnothing$ and $\left\{W_{1}, W_{2}\right\}$ is clustered, it follows that one of these, say $W_{1}$, also fails to intersect $H$ nontrivially. It is clear that the left-hand side must also have value 0 , a contradiction.

If $\mathscr{K}$ is a finite, nonempty collection of closed, convex sets, the closed, transversal characteristic of $\mathscr{K}$ is the function:

$$
\omega_{\mathrm{c}}(\mathscr{K})=\sum_{\substack{\mathscr{A} \subseteq \mathscr{K} \\ \mathscr{A} \neq \varnothing}}(-1)^{|\cdot \mathscr{A}|-1} C\left(\mathrm{cl} \operatorname{conv} \bigcup_{K \in \mathscr{A}} K\right)
$$

Clearly, $\omega_{\mathrm{c}}(\mathscr{K}) \in S_{\mathrm{c}}$.

Theorem 14. Suppose $\mathscr{K}_{1}$ and $\mathscr{K}_{2}$ are finite, nonempty collections of closed, convex sets. Then $\tau\left(\mathscr{K}_{1}\right)=\tau\left(\mathscr{K}_{2}\right)$ if and only if $\omega_{\mathrm{c}}\left(\mathscr{K}_{1}\right)=\omega_{\mathrm{c}}\left(\mathscr{K}_{2}\right)$.

Proof. We observe that if $H$ is a closed half-space, then

$$
\bar{\chi}_{\mathrm{c}}\left(C(H) \cap \omega_{\mathrm{c}}(\mathscr{K})\right)= \begin{cases}0 & \text { if } H \notin \tau(\mathscr{K}), \\ 1 & \text { if } H \in \tau(\mathscr{K}) .\end{cases}
$$

A simple argument shows that $\tau\left(\mathscr{K}_{1}\right)$ and $\tau\left(\mathscr{K}_{2}\right)$ are distinct if and only if there is a closed half-space in one of these sets which is not in the other. 
This theorem enables one to construct an injection of $\hat{L}_{\mathrm{c}}$ into the lattice of complete families of transversals.

We consider next the sublattice, $\Phi_{\mathrm{p}}$, of the lattice of complete families of transversals. If $\mathscr{T}=\tau\left(\left\{P_{1}, \ldots, P_{m}\right\}\right) \in \Phi_{\mathrm{p}}$, where $P_{1}, \ldots$, and $P_{m}$ are convex polytopes, let $\beta(\mathscr{T})=\omega_{c}\left(\left\{P_{1}, \ldots, P_{m}\right\}\right)$. This is a well-defined injection from $\Phi_{\mathrm{p}}$ into $\hat{L}_{c}$, by Theorem 14. It is clear that the image of $\beta$ is a sublattice $\hat{L}_{\mathrm{p}}$ of $\hat{L}_{\mathrm{c}}$, and $\hat{L}_{\mathrm{p}}$ is generated by the characteristic functions of the closed, convex polytopes. In fact, $\beta: \Phi_{\mathrm{p}} \rightarrow \hat{L}_{\mathrm{p}}$ is a (lattice) dual isomorphism.

Recall that, according to Theorem 2 , the restriction of $\tau$ to $\Phi_{p}$ is a dual automorphism. For $F \in \hat{L}_{\mathrm{p}}$, let $\bar{\tau}(F)=\beta \tau \beta^{-1}(F)$. This is a dual automorphism of $\hat{L}_{\mathrm{p}}$.

Let $S_{\mathrm{p}}$ denote the group generated by the characteristic functions of the convex polytopes, so that $S_{\mathrm{p}}$ is a subgroup of $S_{\mathrm{c}}$. Since $S_{\mathrm{o}}$ contains the characteristic function of the relative interior of each convex polytope, and since each nonempty polytope is the disjoint union of the relative interiors of its nonempty faces, it is clear that $S_{\mathrm{p}} \subseteq S_{\mathrm{o}}$, as well.

We use Euler's relation to obtain the connection between $\bar{\chi}_{\mathrm{o}}$ and $\bar{\chi}_{\mathrm{c}}$. If $F$ is the characteristic function of a convex, $k$-dimensional polytope then $F=$ $\Sigma_{K} C(K)$, where the summation extends over all relative interiors of faces of $F$. Applying $\vec{X}_{\mathrm{o}}$ to both sides yields

$$
\begin{aligned}
\bar{\chi}_{\mathrm{o}}(F) & =\sum_{K} \bar{\chi}_{\mathrm{o}}(C(K))=\sum_{K}(-1)^{d-\operatorname{dim} K} \\
& =(-1)^{d}=(-1)^{d} \bar{\chi}_{\mathrm{c}}(F)
\end{aligned}
$$

Since such functions generate $S_{p}$, we have that $\bar{\chi}_{\mathrm{o}}\left|S_{\mathrm{p}}=(-1)^{d} \bar{\chi}_{\mathrm{c}}\right| S_{\mathrm{p}}$.

Lemma 6. Let $\mathscr{K}=\left\{\left\{v_{i}\right\}: 1 \leq i \leq n\right\}$. Then $\omega(\mathscr{K})=(-1)^{\mathrm{dim} K} C($ relint $K)$, where $K=\operatorname{conv}\left\{v_{i}: i \in[n]\right\}$.

Proof. Let $H$ be a closed half-space. Then

$$
\begin{aligned}
\bar{\chi}_{c}(C(H) \cap \omega(\mathscr{K})) & =\sum_{\substack{\Lambda \subseteq[n], \Lambda \neq \varnothing}}(-1)^{|\Lambda|-1} \chi_{c}\left(C(H) \cap \operatorname{conv}\left\{v_{i}: i \in \Lambda\right\}\right) \\
& =\sum_{\Lambda}(-1)^{|\Lambda|-1},
\end{aligned}
$$

where this last summation extends over nonempty sets $\Lambda \subseteq[n]$ such that $\Lambda \cap$ $\left\{i: v_{i} \in H\right\} \neq \varnothing$. This is 1 , if $\left\{i: v_{i} \in H\right\}=[n]$, and 0 , otherwise.

Also we have

$$
\begin{aligned}
\bar{\chi}_{\mathrm{c}}(C & \left.(H) \cap(-1)^{\operatorname{dim} K} C(\text { relint } K)\right) \\
& =(-1)^{\operatorname{dim} K} \bar{\chi}_{\mathrm{c}}(C(H \cap \text { relint } K)) \\
& =(-1)^{\operatorname{dim} K} \bar{\chi}_{\mathrm{c}}(C(\text { int } H \cap \text { relint } K))+\bar{\chi}_{\mathrm{c}}(C(\text { bd } H \cap \text { relint } K))
\end{aligned}
$$


which is 1 , if relint $K \subseteq H$, and 0 , otherwise. By Theorem 12, the result follows.

Theorem 15. The function $\bar{\tau}$ is the restriction of the Sallee-Shephard mapping, $\sigma$, to $\hat{L}_{\mathrm{p}}$.

Proof. If $K=\operatorname{conv}\left\{v_{i}: i \in[n]\right\}$, then $\bar{\tau}(C(K))=(\tau(\{K\}))=\omega\left(\left\{\left\{v_{i}\right\}: i \in[n]\right\}\right)=$ $\sigma(C(K))$. Since $\bar{\tau}$ is a lattice dual isomorphism, $\bar{\tau}: \hat{L}_{\mathrm{p}} \rightarrow \hat{L}_{\mathrm{p}}$, we may view it as a valuation $\bar{\tau}: \hat{L}_{\mathrm{p}} \rightarrow S_{\mathrm{p}}$; it induces a group homomorphism, $\bar{\tau}^{\prime}: S_{\mathrm{p}} \rightarrow S_{\mathrm{p}}$. We have seen that $\bar{\tau}^{\prime}$ and $\sigma$ agree on the characteristic functions of convex polytopes, which generate $S_{\mathrm{p}}$ as a group.

Corollary. If $\mathscr{P}$ and $\mathscr{2}$ are finite, nonempty collections of convex polytopes, then $\tau(\mathscr{P})=\tau(\mathscr{Q})$ if an only if $\omega(\mathscr{P})=\omega(\mathscr{Q})$.

\section{Polarity as a Homomorphism}

In this section we make the easy observation that the normal cone mapping induces an automorphism of the group $S_{\mathrm{c}}$ generated by characteristic functions of closed cones. Analogous results hold for other polarities, e.g., the polar reciprocal mapping.

For $K \subseteq \mathscr{R}^{d}$, let

$$
\operatorname{norm}(K)=\left\{y \in \mathscr{R}^{d}:\langle x, y\rangle \leq 0 \text { for each } x \in K\right\} .
$$

Theorem 16. The mapping $\rho_{0}: C(K) \rightarrow C($ norm $(K))$, for closed, convex sets $K$, has an extension $\rho: S_{\mathrm{c}} \rightarrow S_{\mathrm{c}}$, which is a homomorphism.

Proof. For $f=\sum a_{i} C\left(K_{i}\right) \in S_{\mathrm{c}}$ and $x \in \mathscr{R}^{d}$, define $\rho(f)(x)$ as follows. Choose $\varepsilon>0$ sufficiently small that if there is $y \in K_{i}$ such that $\langle x, y\rangle>0$, then $K_{i} \cap H_{x} \neq \varnothing$, where $H_{x}=\left\{y \in \mathscr{R}^{d}:\langle x, y\rangle \leq-\varepsilon\right\}$. Then the function defined by

$$
\rho(f)(x)=\bar{\chi}(f)-\bar{\chi}\left(H_{x} \cap f\right)=\sum_{K_{1} \cap H_{3}=\varnothing} a_{i}
$$

is clearly a well-defined homomorphism, $\rho: S_{\mathrm{c}} \rightarrow S_{\mathrm{c}}$; and, since $K_{i} \cap H_{x}=\varnothing$ if and only if $x \in \operatorname{norm}\left(K_{i}\right)$ (by our choice of $\varepsilon$ ), it is indeed the case that $\rho(C(K))=$ $\rho_{0}(C(K))$ for closed, convex sets $K$.

Consider the subgroup $M$ of $S_{c}$ generated by the characteristic functions of closed cones. This is isomorphic to the valuation module of the lattice of finite unions of such cones; intersection induces a multiplication such that the resulting ring is isomorphic to the valuation ring of this lattice.

The restriction of $\rho$ to this group is an isomorphism of the ring with intersection as multiplication and the ring with cl conv as multiplication. The image of $\hat{L}_{c} \cap M$ under this mapping is the set of characteristic functions of finite unions of closed cones; we find that $\hat{L}_{\mathrm{c}} \cap M$ is dually isomorphic, as a lattice, to the lattice of finite unions of closed cones. For a related result, see Theorem 1(a) of [12]. 


\section{References}

1. G. Birkhoff, Lattice Theory, Third edn., American Mathematical Society, Providence, RI, 1979.

2. L. Geissinger, Valuations on distributive lattices, 1, II, III, Arch. Math. 24 (1973), 230-239, 337-345, 475-481.

3. H. Groemer, On the Euler characteristic in spaces with a separability property, Math. Ann. 211 (1974), 315-321.

4. H. Groemer, Minkowski addition and mixed volumes, Geom. Dedicat. 6 (1977), 141-163.

5. H. Groemer, On the extension of additive functionals on classes of convex sets, Pacific J. Math. 75 (1978), 397-410.

6. H. Groemer, On rings of sets associated with characteristic functions, preprint.

7. B. Grünbaum, Convex Polytopes, Interscience, New York, 1967.

8. H. Hadwiger, Euler charackteristik und kombinatorische geometrie, J. Reine Angew. Math. 194 (1955), 101-110.

9. P. Hammer, Maximal convex sets, Duke Math. J. 22 (1955), 103-106.

10. R. Jamison, A general duality between the theorems of Caratheodory and Helly, to appear.

11. V. Klee, The Euler characteristic in combinatorial geometry, Amer. Math. Monthly 70 (1963), 119-127.

12. J. Lawrence, Clustered families of convex sets, Houston J. Math., to appear.

13. J. Lawrence, Minkowski rings, to appear.

14. P. McMullen and R. Schneider, Valuations on convex bodies, in Convexity and its applications (P. M. Gruber and J.. M. Wills, eds.), 170-247, Birkhauser, Basel, 1983.

15. R. T. Rockafellar, Convex Analysis, Princeton University Press, Princeton, NJ, 1972.

16. G.-C. Rota, The valuation ring, in Studies in Pure Mathematics (L. Mirsky, ed.), Academic Press, New York, 1971.

17. G. T. Sallee, Polytopes, valuations, and the Euler relation, Canad. J. Math. 20 (1968), 1412-1424.

18. G. C. Shephard, Euler-type relations for convex polytopes, Proc. London Math. Soc. 18 (1968), 597-606.

19. J. Stoer and C. Witzgall, Convexity and Optimization, Springer-Verlag, New York, 1970.

Received June 12, 1985, and in revised form February 23, 1987. 